\title{
Approach to lipid screening as a risk marker for cardiovascular disease in pediatric patients with diabetes
}

\section{Jennifer Rachel Law, Shipra Patel and Anna Spagnoli*}

Division of Pediatric Endocrinology, Department of Pediatrics, University of North Carolina at Chapel Hill, Chapel Hill, NC, USA

\section{Edited by:}

C. L. Acerini, University of

Cambridge, UK

\section{Reviewed by:}

Tim Barrett, University of

Birmingham, UK

Revi P. Mathew, Vanderbilt University

Medical School, USA

*Correspondence:

Anna Spagnoli, Division of Pediatric Endocrinology, University of North

Carolina, 111 Mason Farm Road, CB

7039, Chapel Hill, NC 27599-7039,

USA.

e-mail: spagnoa@med.unc.edu

\begin{abstract}
Cardiovascular disease (CVD) is a well-known complication of diabetes mellitus (DM), and patients with DM are at an increased risk for early onset of CVD. Hyperglycemia is believed to be the primary mediator in premature development of atherosclerosis in patients with DM, but there are also derangements in cholesterol levels and inflammatory markers beyond the explanation of hyperglycemia. Although clinicians often screen for dyslipidemia as part of routine care for children and adolescents with DM, many do not feel comfortable treating this condition. Multiple guidelines exist to help clinicians with the prevention, screening, and treatment of CVD risk factors in pediatric patients with DM, but the guidelines do not always agree on screening intervals or medical treatment. Furthermore, the cost-effectiveness of medication use in this population has not been established. Research has advanced our understanding of the role of other biomarkers and radiologic studies of CVD risk, but these studies do not currently have a place in routine clinical practice. It is evident that the increased CVD risk in pediatric patients with DM is complex in origin and the optimal approach to managing dyslipidemia remains unclear. Therefore, an algorithm designed at the University of North Carolina (UNC), Division of Pediatric Endocrinology, is presented to help guide clinicians through screening and treatment of dyslipidemia in youth with DM.
\end{abstract}

Keywords: diabetes, cardiovascular disease, dyslipidemia, cholesterol, statin

\section{INTRODUCTION}

Cardiovascular disease (CVD) is a well-known complication of diabetes mellitus (DM), and patients with DM are at an increased risk for early onset of CVD. It is evident that atherosclerosis begins in childhood (Berenson et al., 1992; Strong et al., 1999); however, CVD risk factors are most frequently assessed in the adult population. A cross-sectional analysis of the German diabetes documentation and quality management system (DPV) showed that of the 33,488 pediatric patients with type 1 diabetes (T1D) in the system between September 2007 and September 2008, only $26 \%$ had no additional risk factors for CVD [Hemoglobin Alc (HbAlc) $\geq 7.5 \%$, hypertension, dyslipidemia, obesity, and smoking], $39 \%$ had one risk factor, and $35 \%$ had two or more (Schwab et al., 2010)!

Studies have shown that adults with T1D diagnosed in childhood develop more advanced atherosclerosis earlier in life than the general population (Valsania et al., 1991) and have a higher rate of cardiac failure (Savage and Semple, 2010) and shorter survival (Portuese and Orchard, 1995). The primary mediator in atherosclerosis for patients with T1D is hyperglycemia (Kavey et al., 2006). Optimizing glycemic control in patients with DM is generally accepted as the foundation for improving CVD risk; however, the approach to dyslipidemia treatment in the setting of optimal glycemic control is less clear.

With the rise in obesity, insulin resistance, and type 2 diabetes (T2D) in youth, increasing evidence suggests that these patients may be at an even greater risk for CVD than similar aged adolescents with T1D (Wadwa, 2006). Insulin resistance contributes to dyslipidemia as both hepatic synthesis of verylow-density lipoprotein (VLDL-C) is enhanced and peripheral action of lipoprotein lipase is decreased, leading to increases in low-density lipoprotein (LDL-C) and triglyceride (TG) levels (Kavey et al., 2006; Savage and Semple, 2010). These derangements as well as low HDL-C levels have been shown to correlate with the degree of atherosclerosis in children (NCEP Expert Panel on Blood Cholesterol Levels in Children and Adolescents, 1992).

Much research has been done to identify biomarkers and noninvasive radiological findings that suggest either existing CVD or CVD risk. While measurements such as carotid intima media thickness (cIMT) and C-reactive protein (CRP) are used as markers of CVD in adults, their utility in pediatrics is debatable. Beyond the use of these studies in the research field, their role in pediatric clinical practice has not been established.

\section{CURRENT CHOLESTEROL GUIDELINES}

Many guidelines have been developed to help clinicians manage patients with DM and dyslipidemia (see Table 1). In this age of evidence-based medicine, it is disappointing that the recommendations must be frequently based on individual expert opinions and clinical experience. Researchers have had difficulty obtaining prospective longitudinal data and experimenting with drugs and interventions in children, thereby limiting the evidence available. 
Table 1 | Cholesterol guidelines overview*.

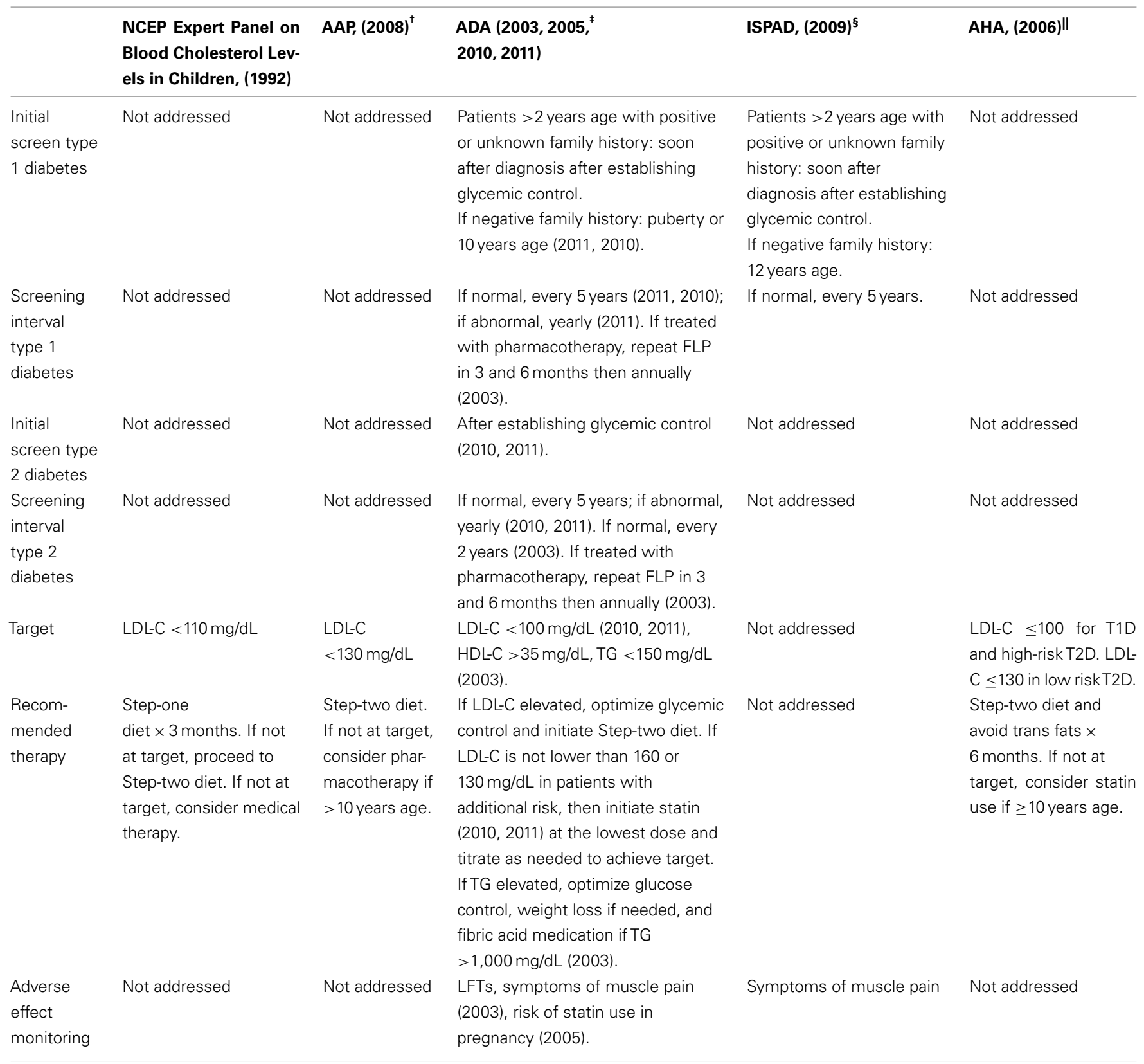

${ }^{*}$ Guidelines are listed in the order of which they were discussed in the text. The order does not indicate author preference. ${ }^{+}$Daniels et al. (2008). ${ }^{*}$ Silverstein et al. (2005). ${ }^{5}$ Donaghue et al. (2009). "Kavey et al. (2006).

In 1992, the National Cholesterol Education Program (NCEP) published guidelines for approaching dyslipidemia in children; however, dyslipidemia management specifically in patients with DM is not addressed. These guidelines describe a two-step dietary approach for elevated LDL-C treatment prior to medical therapy. Step-one of this approach entails a low fat diet $(\leq 30 \%$ of total calories from total fat, $<10 \%$ of total calories from saturated fatty acids, $\leq$ of total calories from polyunsaturated fatty acids), $<300 \mathrm{mg} /$ day of cholesterol, carbohydrates accounting for $55 \%$ of total calories, and protein accounting for $15-20 \%$ of total calories. Patients should practice this step-one diet for 3 months. If the patients do not achieve target LDL-C levels of $<110 \mathrm{mg} / \mathrm{dL}$, then patients should proceed to the step-two diet, in which saturated fatty acids now comprise $<7 \%$ of total calories and cholesterol is limited to $<200 \mathrm{mg} /$ day (NCEP Expert Panel on Blood Cholesterol Levels in Children and Adolescents, 1992).

The Dietary Intervention Study in Children demonstrated that after 3 years of follow-up, there was a significant difference in LDL-C levels after implementing a step-two diet compared to the 
usual care group with no adverse effects on growth and development (Kwiterovich, 2001). If LDL-C levels are not at target after practicing step-two of the dietary changes, then medical therapy should be considered in children aged $\geq 10$ years with LDL-C $\geq 190 \mathrm{mg} / \mathrm{dL}$ or LDL-C $>160 \mathrm{mg} / \mathrm{dL}$ with either a positive family history of CVD before age 55 years or presence of $\geq 2$ risk factors (cigaret smoking, hypertension, HDL-C $<35 \mathrm{mg} / \mathrm{dL}$, body mass index $(\mathrm{BMI}) \geq 95 \%$ percentile, DM, and physical inactivity; NCEP Expert Panel on Blood Cholesterol Levels in Children and Adolescents, 1992). The 2008 American Academy of Pediatrics guidelines entitled "Lipid Screening and Cardiovascular Health in Childhood" recommended consideration of pharmacotherapy in patients with DM and LDL-C $\geq 130 \mathrm{mg} / \mathrm{dL}$ if $\geq 10$ years of age or older (Daniels et al., 2008).

According to the 2010 and 2011 American Diabetes Association (ADA) "Standards of Medical Care in Diabetes," patients with T1D who are $>2$ years of age with a family history of hypercholesterolemia [total cholesterol (TC) $>240 \mathrm{mg} / \mathrm{dL}$ ], a cardiovascular event before age 55 years, or unknown family history should be screened with a fasting lipid profile (FLP) soon after diagnosis and glycemic control has been achieved. If family history is not concerning, then lipid screening should begin at puberty or $\geq 10$ years of age. If DM diagnosis occurs after puberty, then a FLP should be performed after establishing glycemic control. If LDL-C levels are $<100 \mathrm{mg} / \mathrm{dL}$, then the lipid profile should be repeated every 5 years (American Diabetes Association, 2010, 2011). According to the 2011 guidelines, if the profile is abnormal, it should be repeated yearly (American Diabetes Association, 2011). However, it is not clear if this screening interval is different for patients treated with cholesterol-lowering medications. In patients with T2D, FLP should be obtained at the time of diagnosis. After diagnosis of T2D, both ADA standards state that guidelines are "similar to those for youth with type 1 diabetes," suggesting that screening occur after establishing glycemic control then every 5 years if LDL-C is $<100 \mathrm{mg} / \mathrm{dL}$ or every 1 year if LDL-C is $>100 \mathrm{mg} / \mathrm{dL}$ (American Diabetes Association, 2010, 2011). These 2010 and 2011 standards differ somewhat from the ADA's 2003 consensus statement on "Management of Dyslipidemia in Children and Adolescents with Diabetes." Previously, the recommended screening interval in patients with T2D with normal lipid profiles was every 2 years (American Diabetes Association, 2003). With the high prevalence of comorbidities in patients with T2D and the insidious nature of symptom-onset leading to possibly years prior to diagnosis, it seems that the more frequent monitoring of lipid profiles would be favored.

For patients diagnosed with dyslipidemia from FLPs, the initial recommended therapy according to the 2010 and 2011 ADA guidelines should include optimizing glycemic control and using step-two of the NCEP diet as described above. After 10 years of age, if lifestyle changes have not lowered the LDL-C to $\leq 160$ or $\leq 130 \mathrm{mg} / \mathrm{dL}$ in patients with additional CVD risk factors, then a statin is recommended for therapy to achieve a LDL-C of $<100 \mathrm{mg} / \mathrm{dL}$ (American Diabetes Association, 2010, 2011). The ADA recommended that statins should be started at the lowest dose available and titrated up as needed. Periodic monitoring of liver function tests (LFTs) is necessary in statin use, and medications should be stopped if LFTs exceed three times the upper limit of normal. Statins should also be discontinued if persistent muscle pain occurs, but routine creatinine phosphokinase monitoring was not felt to be helpful (American Diabetes Association, 2003).

Though the Lovastatin in Adolescent Male study has shown statins to decrease LDL-C levels without adverse effects on growth and development in adolescent males over 7 years when compared to usual care, the ADA has recognized that long-term safety and efficacy data is not available (Kwiterovich, 2001; American Diabetes Association, 2010, 2011). In the 2005 guidelines entitled "Care of Children and Adolescents with Type 1 Diabetes" the ADA also raised concerns about the use of statins in sexually active females as these drugs are not approved in pregnant individuals (Silverstein et al., 2005).

The 2003 statement further describes optimal HDL-C levels of $>35 \mathrm{mg} / \mathrm{dL}$ and TG levels $<150 \mathrm{mg} / \mathrm{dL}$. Elevated TG levels should be treated by optimizing glucose control, weight loss if needed, and using fibric acid medication if TG levels $\geq 1,000 \mathrm{mg} / \mathrm{dL}$. For patients on medical treatment for dyslipidemia, the ADA recommends repeating a FLP 3 and 6 months after therapy initiation, and then annually once treatment goals are achieved (American Diabetes Association, 2003).

The International Society for Pediatric and Adolescent Diabetes published clinical practice guidelines in 2009 similar to the ADA guidelines from 2010 to 2011 with the exception that initial lipid screening in patients without a family history of CVD and without additional risk factors should begin at age 12 years. The recommended interventions for dyslipidemia included improvement of glycemic control and statin use. The authors also recognized that long-term safety of statins has not yet been established (Donaghue et al., 2009).

In the 2006 scientific statement regarding CVD risk reduction in high-risk pediatric patients, the American Heart Association (AHA) identified T1D and T2D as one of eight conditions at risk for premature atherosclerosis beginning in childhood. The evidence for early coronary disease further stratified T1D as part of the high-risk tier I category and T2D as part of the moderate risk tier II category. However, patients with T2D are usually classified as high risk in tier I because of the presence of at least two other comorbidities (dyslipidemia, smoking, CVD $\leq 55$ years of age for first degree male relatives and $\leq 65$ years of age for first degree female relatives, hypertension, overweight/obese, physically inactive). Children within tier I should be approached as having a "coronary heart disease equivalent." The AHA recommends that those patients in the tier 1 category obtain LDL-C $\leq 100 \mathrm{mg} / \mathrm{dL}$ and those within the tier II category obtain LDL-C $\leq 130 \mathrm{mg} / \mathrm{dL}$. In order to reach these cholesterol levels, the first recommended step is to initiate the NCEP step-two diet under the guidance of a nutritionist with the addition of avoidance of trans fats for 6 months. If LDL-C goals are not achieved, then statin therapy should be considered if the child is older than 10 years of age (Kavey et al., 2006). The AHA guidelines were endorsed by the American Academy of Pediatrics.

Aside from the 2003 ADA consensus statement that addresses hypertriglyceridemia, there are no other official guidelines for the approach to TG or HDL-C management. The Pittsburgh Epidemiology of Diabetes Complications Study evaluated 10 years of mortality and morbidity data related to T1D. In this study of nearly 
600 patients, risk ratios were calculated between cholesterol levels and mortality, coronary artery disease, and overt nephropathy. Based on risk ratios of at least 1.8, the following cholesterol goal levels were recommended by the researchers: LDL-C $<100 \mathrm{mg} / \mathrm{dL}$, HDL-C $>45 \mathrm{mg} / \mathrm{dL}$, TG $<150 \mathrm{mg} / \mathrm{dL}$ (Orchard et al., 2001).

Recent studies have suggested that additional lipid parameters may be useful in the assessment and management of youth with DM. The SEARCH for Diabetes in Youth study found an association between glycemic control and fasting lipid concentrations in subjects with T1D, as those with a HbA1c $<7.5 \%$ had similar TC and LDL-C levels but lower TG and higher HDL-C levels compared to healthy controls, while the patients with $\mathrm{HbAlc} \geq 7.5 \%$ had elevated TC, LDL-C, non-HDL-C, and HDL-C levels. Interestingly, the subjects with DM (even those in optimal control) had significantly higher apolipoprotein B (Apo B) and a greater number of small dense LDL-C particles than the control subjects, suggesting that the lipids in patients with DM in some ways may be more atherogenic. Small dense LDL-C, which contains less cholesterol per particle and tends to become oxidized, has been shown to be an independent predictor of CVD (Guy et al., 2009). In patients with DM, LDL-C particle number may be higher at any given LDL-C concentration, likely because of the increased percentage of small dense LDL-C particles (Vijayaraghavan, 2010). Apo B has also been shown to be an independent predictor of CVD and may provide a better estimate of LDL-C particle number than LDL-C or nonHDL-C in patients with insulin resistance and DM (Ganda, 2009). Therefore, it may be useful for future guidelines to incorporate other lipid parameters as additional goals of therapy. Overall, it appears that there are both gaps and discrepancies among the different clinical guidelines, making it difficult for providers who are seeking specific guidelines for their patients with DM.

\section{COST OF DYSLIPIDEMIA}

The incidence rates of both T1D and T2D are rising, and with them the burden of their complications. As with much other data in pediatric DM, cost-analysis data does not exist for the treatment of dyslipidemia beginning in childhood. Adult literature shows that several European cost-effectiveness studies based on the "Collaborative Atorvastatin Diabetes Study" have determined atorvastatin use in adults to be cost-effective to various degrees depending on the country in which the drug is to be used when compared to no treatment in the prevention of CVD (Raikou et al., 2007; Lafuma et al., 2008; Annemans et al., 2010).

A systematic review of interventions in adults for preventing and controlling DM demonstrated that statin use was "very cost-effective" in the secondary prevention of CVD, while those strategies described as "cost-effective" included the primary prevention of CVD for patients with T2D and hyperlipidemia, intensive insulin treatment for people with T1D compared with conventional glycemic control, and intensive glycemic control by a Diabetes Prevention Program type of intensive lifestyle intervention (ILI) in people with newly diagnosed T2D compared with conventional glycemic control. Surprisingly, intensive glycemic control for all U.S. residents with T2D diagnosed at age $\geq 25$ years compared with usual care was only "marginally cost-effective" (Li et al., 2010). One would presume that glycemic control would be the most cost-effective since it is considered to be the cornerstone of dyslipidemia treatment. The AHEAD study examined the cost-savings of an ILI regimen compared to a DM support and education system in adults with T2D. In this study, the ILI group improved their lipid profiles, decreased medication use, and saved \$40 per month in medication costs (Redmon et al., 2010).

\section{OTHER CVD RISK MARKERS}

Other biomarkers and radiologic testing are frequently performed for research purposes to better understand the pathophysiological course of CVD, but what is the role of this testing in routine clinical practice? Before implementing these tests into routine care, normative values must be established in the healthy pediatric population for each of these tests.

Carotid intima media thickness has been used in the adult population to assess the degree of CVD risk due to atherosclerosis. This practice is based on studies suggesting that cIMT correlates with risk of cardiovascular events (de Groot et al., 2004). One longitudinal study of patients with T1D over a 2-year period showed that of the patients who had an elevated cIMT at baseline, those who had worsening of cIMT also had a higher HbAlc, though there were no significant differences in lipid profile, systolic blood pressure, or BMI measurements (Dalla Pozza et al., 2010). Although studies have also revealed a thicker cIMT in patients with T1D compared to those without DM, conflicting data exists regarding whether cIMT is associated with DM duration (Atabek et al., 2006; Dalla Pozza et al., 2007; Rabago Rodriguez et al., 2007; Margeirsdottir et al., 2010).

One study from the International Childhood Cardiovascular Cohort (iC3) Consortium examined the association in healthy subjects between cholesterol, BMI, and systolic blood pressure among different childhood age groups and cIMT in adulthood. This study showed that TC level was associated with cIMT in adulthood beginning at age 12, systolic blood pressure was associated with cIMT beginning at age 6 , and BMI was associated with cIMT beginning at age 9 (Juonala et al., 2006, 2010). A similar study using data from the Bogalusa Heart Study, Young Finns Study, and Childhood Determinants of Adult Health Study found that adolescents with elevated LDL-C had an increased risk of high cIMT if LDL-C did not normalize by adulthood. Both groups with persistent low HDL-C and with low HDL-C that normalized by adulthood had an increased risk of high cIMT (Magnussen et al., 2009). While these CVD risk factors in children predict cIMT in adulthood, what does cIMT in childhood indicate? Furthermore, to what extent are cIMT derangements found in childhood reversible through early interventions?

Many studies have examined the normative values for cIMT in healthy children. Some studies suggest that cIMT is positively associated with age (Ishizu et al., 2004; Bohm et al., 2009), while other studies do not find this association (Sass et al., 1998). Gender differences were appreciated in one major study by Bohm et al. (2009), but not found in another large study in a similar population (Jourdan et al., 2005). Clearly there is disagreement in cIMT measurements even in similar populations and none of the standards proposed would be generalizable to a clinic with patients of mixed ethnic and racial backgrounds. It is also likely that cIMT methods vary across institutions and may be evaluator-dependent. 
Other radiologic measures for assessing vascular function include arterial compliance, stiffness, and distensibility. Two studies (one adult, one pediatric) have shown that patients with T1D have lower arterial compliance and distensibility when compared to healthy controls; nevertheless, exercise was shown to improve these measures in both groups, but not to the same levels as healthy controls (Mason et al., 2006; Trigona et al., 2010). Although some evidence suggests that patients with T1D have reduced arterial compliance and distensibility as well as increased arterial stiffness, some studies disagree (Galler et al., 2010; Margeirsdottir et al., 2010). A scientific statement by the AHA in 2009 regarding the use of measurements of cIMT, coronary artery calcification by computed tomography, arterial stiffness, arterial distensibility, and arterial dilation concluded that "Additional data are needed before these methods can be adopted in clinical evaluation" (Urbina et al., 2009).

Many researchers focus on the role of inflammation in CVD development. Pediatric patients with T1D have been shown to have increased inflammatory markers even with acceptable glycemic control (Snell-Bergeon et al., 2010). In two studies of children and young adults with T1D, elevated serum levels of inflammatory markers such as CRP were associated with higher cIMT measurements, suggesting an increased risk of CVD (Hayaishi-Okano et al., 2002; Atabek et al., 2006). However, another study showed that while CRP in childhood was weakly associated with CRP levels in adulthood, it was not associated with cIMT in adulthood (Juonala et al., 2006). It is important to note that standard CRP normative ranges are generally not reported in the pediatric population (McNeal et al., 2009).

A recent study showed that inflammatory markers such as IL-6 and fibrinogen were elevated in youth with T1D, regardless of their weight status or glycemic control. High sensitivity CRP (hs-CRP) was also found to be higher in the patients with worsening glycemic control. Moreover, elevations in these inflammatory markers were associated with a more atherogenic lipid profile (higher TC, LDLC, and Apo B, but lower HDL-C; Snell-Bergeon et al., 2010). Other biomarkers that reflect endothelial cell dysfunction have been studied very little in children. One study found that patients with T1D had elevated levels of intercellular adhesion molecule (ICAM), nitric oxide (NO), and malondialdehyde (MDA) when compared to healthy controls, and those with poor glycemic control also had higher levels of vascular endothelial growth factor (VEGF; Seckin et al., 2006).

One routine measurement that is used to screen for nephropathy may have a new role in predicting CVD risk: urine microalbumin. Adult studies have shown $24 \mathrm{~h}$ urine albumin levels to predict increased risk for CVD in both patients with T1D and T2D (Deckert et al., 1996; Miettinen et al., 1996; Zhan et al., 2009). While it is exciting to think of the possibility of using one tool to screen for two common complications of DM, little is known about the predictive value of microalbuminuria for CVD in the pediatric population.

\section{CHOLESTEROL ALGORITHM}

At the UNC, Division of Pediatric Endocrinology, we have compiled the major guidelines and consensus statements regarding dyslipidemia management in pediatric patients with DM into an algorithm designed to help our providers practically apply the latest recommendations regarding lipid screening and treatment (see Figure 1). We are in the process of collecting data to evaluate its effectiveness. The algorithm underwent review by the UNC Pediatric Endocrinology Division members prior to implementation and has been approved by the Division for use in the UNC Pediatric Endocrinology diabetes clinic.

Of note, our screening interval for patients with T2D who have a history of normal lipids is based on the ADA's guidelines from 2003, because we felt that their high prevalence of comorbidities and increased risk for CVD warrants closer monitoring of lipids. Our initial screen is a non-FLP (TC, HDL-C, and non-HDL-C) because most of our patients do not come to appointments fasting. If our patients have been fasting for more than $8 \mathrm{~h}$, we begin with a FLP (TC, LDL, TG, HDL-C). We rely on the UNC laboratory reference range for distinguishing normal from abnormal values for non-FLPs. These ranges may differ at other institutions. We rescreen patients with normal non-FLPs the same as the 2003 ADA recommended screening intervals for patients with normal FLPs. If the non-FLP is abnormal, we then arrange a FLP for further evaluation. Our algorithm is then based on the 2011 ADA guidelines unless a different set of guidelines addresses a topic not addressed by the 2011 ADA guidelines. Because there has been little guidance besides lifestyle modifications in the approach to improving low HDL-C or elevated TG levels, we have not addressed these cholesterol derangements in our algorithm. Our diabetes care providers have felt that this tool has been a useful reference for when to screen for and how to treat dyslipidemia in the UNC pediatric diabetes population.

\section{CONCLUSION}

Cholesterol screening is not universal among pediatricians, and despite compelling data that pediatric patients with DM are at increased risk for CVD, clinicians often do not feel comfortable prescribing dyslipidemic medications because of concerns for long-term medication safety. Additionally, there is no longitudinal data to show that early dyslipidemia treatment improves CVD outcome. One prescription claims data study of commercially insured children in the United States from 2004 to 2007 showed that the prevalence of dyslipidemia medication prescriptions was 0.2 per 1000 youths, of which statins accounted for 56.9\%. During the study period, dyslipidemia prescription claims dropped by 14 $20 \%$ overall except in 16-18 year old females whose use increased by $14 \%$; however, insurance claims for statins decreased by $22.9 \%$ overall (Liberman et al., 2009).

Current clinical guidelines addressing dyslipidemia in youth with DM have been derived from expert opinions or extrapolated from the adult literature. There are limited recommendations regarding treatment of high TG or low HDL-C, and the most current guidelines do not clearly delineate any differences in the approach to pediatric T1D versus T2D. Because recent studies have revealed a greater number of CVD risk factors in youth with T2D compared to those with T1D, guidelines for pediatric T2D may need to be more aggressive.

Further studies are needed to evaluate the long-term safety and effectiveness of statins and other cholesterol-lowering medications in children with DM, and to determine whether early treatment 


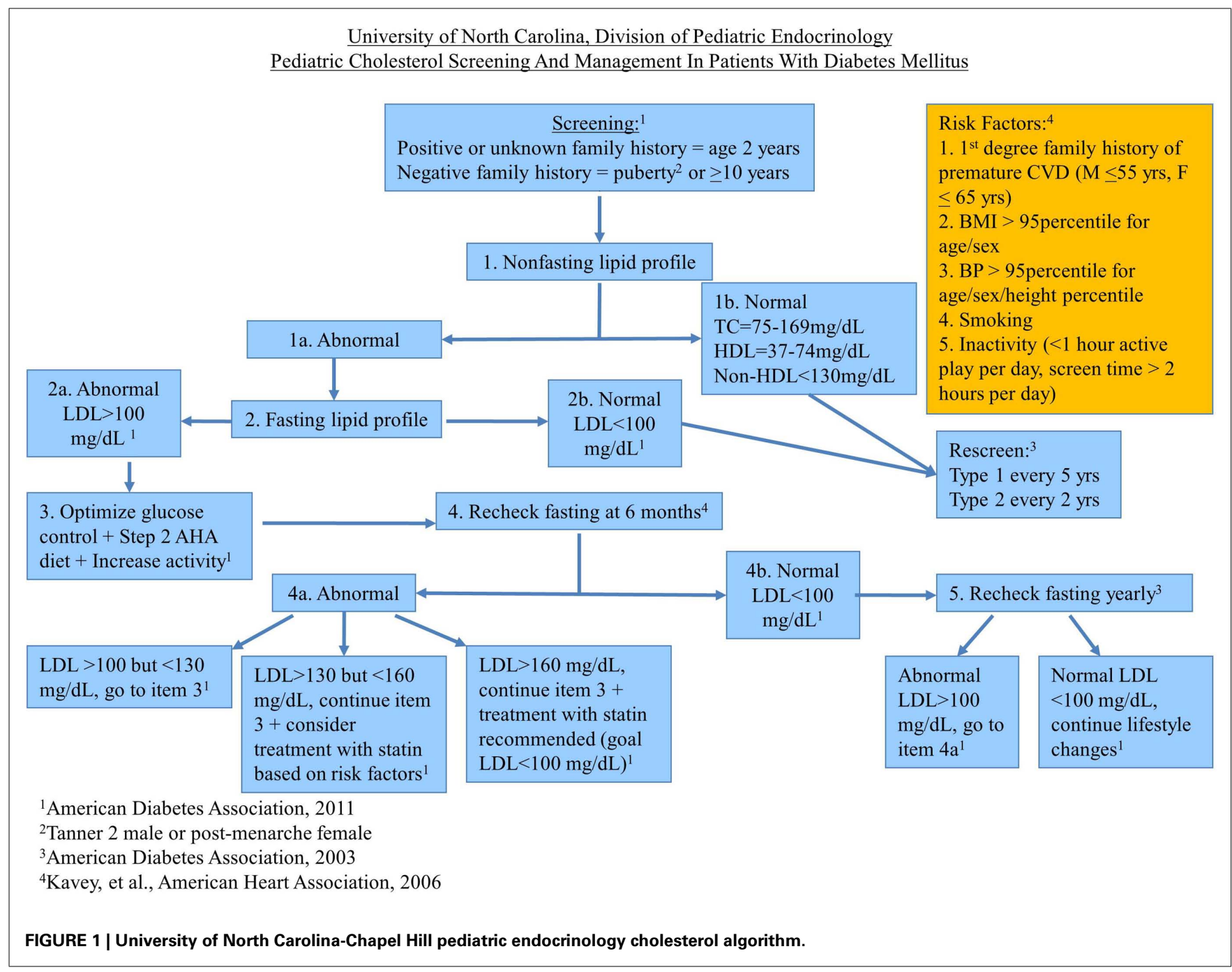

significantly reduces CVD in adulthood. Additional research is also needed to explore the clinical usefulness of various biomarkers and radiographic measurements. Though the optimal approach to managing dyslipidemia in pediatric patients with DM remains unclear, it can be confidently stated that they are at high risk for CVD, and we, as the medical and scientific community, must strive to find the optimal prevention, screening, and treatment regimen.

\section{REFERENCES}

American Diabetes Association. (2003). Management of dyslipidemia in children and adolescents with diabetes. Diabetes Care 26, 2194-2197. American Diabetes Association. (2010). Standards of medical care in diabetes - 2010. Diabetes Care 33, S11-S61.

American Diabetes Association. (2011). Standards of medical care in diabetes - 2011. Diabetes Care 34, S11-S61.

Annemans, L., Marbaix, S., Webb, K., Van Gaal, L., and Scheen, A. (2010).
Cost effectiveness of atorvastatin in litus: a pharmacoeconomic analysis of the collaborative atorvastatin diabetes study in the belgian population. Clin. Drug Investig. 30, 133-142.

Atabek, M. E., Pirgon, O., Kurtoglu, S., and Imamoglu, H. (2006). Evidence for an association between type 1 diabetes and premature carotid atherosclerosis in childhood. Pediatr. Cardiol. 27, 428-433.

Berenson, G. S., Wattigney, W. A., Tracy, R. E., Newman, W. P. III, Srinivasan, patients with type 2 diabetes mel-

\section{ACKNOWLEDGMENTS}

The work above was supported by Award Number T32DK007129 from the National Institute of Diabetes and Digestive and Kidney Diseases. The content is solely the responsibility of the authors and does not necessarily represent the official views of the National Institute of Diabetes and Digestive and Kidney Diseases or the National Institutes of Health.

S. R., Webber, L. S., Dalferes, E. R. Jr., and Strong, J. P. (1992). Atherosclerosis of the aorta and coronary arteries and cardiovascular risk factors in persons aged 6 to 30 years and studied at necropsy (the bogalusa heart study). Am. J. Cardiol. 70, 851-858.

Bohm, B., Hartmann, K., Buck, M., and Oberhoffer, R. (2009). Sex differences of carotid intima-media thickness in healthy children and adolescents. Atherosclerosis 206, 458-463.

Dalla Pozza, R., Bechtold, S., Bonfig, W., Putzker, S., Kozlik-Feldmann, R., Netz, H., and Schwarz, H. P. (2007).
Age of onset of type 1 diabetes in children and carotid intima medial thickness. J. Clin. Endocrinol. Metab. 92, 2053-2057.

Dalla Pozza, R., Netz, H., Schwarz, H. P., and Bechtold, S. (2010). Subclinical atherosclerosis in diabetic children: results of a longitudinal study. Pediatr. Diabetes 11, 129-133.

Daniels, S. R., Greer, F. R., and Committee on Nutrition. (2008). Lipid screening and cardiovascular health in childhood. Pediatrics 122, 198-208. 
de Groot, E., Hovingh, G. K., Wiegman, A., Duriez, P., Smit, A. J., Fruchart, J. C., and Kastelein, J. J. (2004). Measurement of arterial wall thickness as a surrogate marker for atherosclerosis. Circulation 109, III33-III38.

Deckert, T., Yokoyama, H., Mathiesen, E., Ronn, B., Jensen, T., FeldtRasmussen, B., Borch-Johnsen, K., and Jensen, J. S. (1996). Cohort study of predictive value of urinary albumin excretion for atherosclerotic vascular disease in patients with insulin dependent diabetes. $\mathrm{Br}$. Med. J. 312, 871-874.

Donaghue, K. C., Chiarelli, F., Trotta, D., Allgrove, J., and Dahl-Jorgensen, K. (2009). Microvascular and macrovascular complications associated with diabetes in children and adolescents. Pediatr. Diabetes 10, 195-203.

Galler, A., Heitmann, A., Siekmeyer, W., Gelbrich, G., Kapellen, T., Kratzsch, J., and Kiess, W. (2010). Increased arterial stiffness in children and adolescents with type 1 diabetes: No association between arterial stiffness and serum levels of adiponectin. Pediatr. Diabetes 11, 38-46.

Ganda, O. P. (2009). Refining lipoprotein assessment in diabetes: apolipoprotein B makes sense. Endocr. Pract. 15, 370-376.

Guy, J., Ogden, L., Wadwa, R. P., Hamman, R. F., Mayer-Davis, E. J., Liese, A. D., D'Agostino, R., Marcovina, S., and Dabelea, D. (2009). Lipid and lipoprotein profiles in youth with and without type 1 diabetes. Diabetes Care 32, 416-420.

Hayaishi-Okano, R., Yamasaki, Y., Katakami, N., Ohtoshi, K., Gorogawa, S., Kuroda, A., Matsuhisa, M., Kosugi, K., Nishikawa, N., Kajimoto, Y., and Hori, M. (2002). Elevated C-reactive protein associates with early-stage carotid atherosclerosis in young subjects with type 1 diabetes. Diabetes Care 25, 1432-1438.

Ishizu, T., Ishimitsu, T., Yanagi, H., Seo, Y., Obara, K., Moriyama, N., Watanabe, S., and Yamaguchi, I. (2004). Effect of age on carotid arterial intima-media thickness in childhood. Heart Vessels 19, 189-195.

Jourdan, C., Wuhl, E., Litwin, M., Fahr, K., Trelewicz, J., Jobs, K., Schenk, J. P., Grenda, R., Mehls, O., Troger, J., and Schaefer, F. (2005). Normative values for intima-media thickness and distensibility of large arteries in healthy adolescents. J. Hypertens. 23, 1707-1715.

Juonala, M., Magnussen, C. G., Venn, A., Dwyer, T., Burns, T. L., Davis, P. H., Chen, W., Srinivasan, S. R.,
Daniels, S. R., Kahonen, M., Laitinen, T., Taittonen, L., Berenson, G. S., Viikari, J. S., and Raitakari, O. T. (2010). Influence of age on associations between childhood risk factors and carotid intimamedia thickness in adulthood: The cardiovascular risk in young finns study, the childhood determinants of adult health study, the bogalusa heart study, and the muscatine study for the international childhood cardiovascular cohort (i3C) consortium. Circulation 122, 2514-2520.

Juonala, M., Viikari, J. S., Ronnemaa, T., Taittonen, L., Marniemi, J., and Raitakari, O. T. (2006). Childhood C-reactive protein in predicting CRP and carotid intima-media thickness in adulthood: The cardiovascular risk in young finns study. Arterioscler. Thromb. Vasc. Biol. 26, 1883-1888.

Kavey, R. E., Allada, V., Daniels, S. R., Hayman, L. L., McCrindle, B. W., Newburger, J. W., Parekh, R. S., Steinberger, J., American Heart Association Expert Panel on Population, and Prevention Science, American Heart Association Council on Cardiovascular Disease in the Young, American Heart Association Council on Epidemiology, and Prevention, American Heart Association Council on Nutrition, Physical Activity, and Metabolism, American Heart Association Council on High Blood Pressure Research, American Heart Association Council on Cardiovascular Nursing, American Heart Association Council on the Kidney in Heart Disease, and Interdisciplinary Working Group on Quality of Care, and Outcomes Research. (2006). Cardiovascular risk reduction in high-risk pediatric patients: A scientific statement from the American Heart Association expert panel on population and prevention science; the councils on cardiovascular disease in the young, epidemiology and prevention, nutrition, physical activity and metabolism, high blood pressure research, cardiovascular nursing, and the kidney in heart disease; and the interdisciplinary working group on quality of care and outcomes research: endorsed by the American Academy of Pediatrics. Circulation 114, 2710-2738.

Kwiterovich, P. O. Jr. (2001). Safety and efficacy of treatment of children and adolescents with elevated low density lipoprotein levels with a step two diet or with lovastatin. Nutr. Metab. Cardiovasc. Dis. 11(Suppl. 5), 30-34.
Lafuma, A., Colin, X., and Solesse, A. (2008). Cost-effectiveness of atorvastatin in the prevention of cardiovascular events in diabetic patients: a French adaptation of CARDS. Arch. Cardiovasc. Dis. 101, 327-332.

Li, R., Zhang, P., Barker, L. E., Chowdhury, F. M., and Zhang, X. (2010). Cost-effectiveness of interventions to prevent and control diabetes mellitus: a systematic review. Diabetes Care 33, 1872.

Liberman, J. N., Berger, J. E., and Lewis, M. (2009). Prevalence of antihypertensive, antidiabetic, and dyslipidemic prescription medication use among children and adolescents. Arch. Pediatr. Adolesc. Med. 163, 357-364.

Magnussen, C. G., Venn, A., Thomson, R., Juonala, M., Srinivasan, S. R., Viikari, J. S., Berenson, G. S., Dwyer, T., and Raitakari, O. T. (2009). The association of pediatric low- and high-density lipoprotein cholesterol dyslipidemia classifications and change in dyslipidemia status with carotid intimamedia thickness in adulthood evidence from the cardiovascular risk in young finns study, the bogalusa heart study, and the CDAH (childhood determinants of adult health) study. J. Am. Coll. Cardiol. 53, 860-869.

Margeirsdottir, H. D., Stensaeth, K. H., Larsen, J. R., Brunborg, C., and DahlJorgensen, K. (2010). Early signs of atherosclerosis in diabetic children on intensive insulin treatment: a population-based study. Diabetes Care 33, 2043-2048.

Mason, N. J., Jenkins, A. J., Best, J. D., and Rowley, K. G. (2006). Exercise frequency and arterial compliance in non-diabetic and type 1 diabetic individuals. Eur. J. Cardiovasc. Prev. Rehabil. 13, 598-603.

McNeal, C. J., Wilson, D. P., Christou, D., Bush, R. L., Shepherd, L. G., Santiago, J., and Wu, G. Y. (2009). The use of surrogate vascular markers in youth at risk for premature cardiovascular disease. J. Pediatr. Endocrinol. Metab. 22, 195-211.

Miettinen, H., Haffner, S. M., Lehto, S., Ronnemaa, T., Pyorala, K. and Laakso, M. (1996). Proteinuria predicts stroke and other atherosclerotic vascular disease events in nondiabetic and non-insulindependent diabetic subjects. Stroke 27, 2033-2039.

NCEP Expert Panel on Blood Cholesterol Levels in Children and Adolescents. (1992). National cholesterol education program (NCEP): Highlights of the report of the expert panel on blood cholesterol levels in children and adolescents. Pediatrics 89, 495-501.

Orchard, T. J., Forrest, K. Y., Kuller, L. H., and Becker, D. J. (2001). Lipid and blood pressure treatment goals for type 1 diabetes: 10-year incidence data from the Pittsburgh epidemiology of diabetes complications study. Diabetes Care 24, 1053-1059.

Portuese, E., and Orchard, T. (1995). "Mortality in insulin-dependent diabetes," in Diabetes in America, 2nd Edn, eds M. I. Harris, C. C. Cowie, M. P. Stern, E. J. Boyko, G. Reiber, and P. H. Bennett (Bethesda, MD National Diabetes Data Group of the National Institute of Diabetes and Digestive, and Kidney Diseases, NIH Publication), 221-232.

Rabago Rodriguez, R., Gomez-Diaz, R. A., Tanus Haj, J., Avelar Garnica, F. J., Ramirez Soriano, E., Nishimura Meguro, E., Aguilar-Salinas, C. A., and Wacher, N. H. (2007). Carotid intima-media thickness in pediatric type 1 diabetic patients. Diabetes Care 30, 2599-2602.

Raikou, M., McGuire, A., Colhoun, H. M., Betteridge, D. J., Durrington, P. N., Hitman, G. A., Neil, H. A., Livingstone, S. J., Charlton-Menys, V., Fuller, J. H., and CARDS Investigators. (2007). Cost-effectiveness of primary prevention of cardiovascular disease with atorvastatin in type 2 diabetes: Results from the collaborative atorvastatin diabetes study (CARDS). Diabetologia 50, 733-740.

Redmon, J. B., Bertoni, A. G., Connelly, S., Feeney, P. A., Glasser, S. P., Glick, H., Greenway, F., Hesson, L. A., Lawlor, M. S., Montez, M., Montgomery, B., and Look AHEAD Research Group. (2010). Effect of the look AHEAD study intervention on medication use and related cost to treat cardiovascular disease risk factors in individuals with type 2 diabetes. Diabetes Care 33, 1153-1158.

Sass, C., Herbeth, B., Chapet, O., Siest, G., Visvikis, S., and Zannad, F (1998). Intima-media thickness and diameter of carotid and femoral arteries in children, adolescents and adults from the stanislas cohort: effect of age, sex, anthropometry and blood pressure. J. Hypertens. 16 1593-1602.

Savage, D. B., and Semple, R. K. (2010). Recent insights into fatty liver, metabolic dyslipidaemia and their links to insulin resistance. Curr. Opin. Lipidol. 21, 329-336. 
Schwab, K. O., Doerfer, J., Marg, W., Schober, E., and Holl, R. W. (2010). Characterization of 33488 children and adolescents with type 1 diabetes based on the gender specific increase of cardiovascular risk factors. Pediatr. Diabetes 11, 357-363.

Seckin, D., Ilhan, N., Ilhan, N., and Ertugrul, S. (2006). Glycaemic control, markers of endothelial cell activation and oxidative stress in children with type 1 diabetes mellitus. Diabetes Res. Clin. Pract. 73, 191-197.

Silverstein, J. J., Klingensmith, G. J., Copeland, K., Plotnick, L., Kaufman, F., Laffel, L., Deeb, L., Grey, M., Anderson, B., Holzmeister, L. A., and Clark, N. (2005). Care of children and adolescents with type 1 diabetes: a statement of the American Diabetes Association. Diabetes Care 28, 186-212.

Snell-Bergeon, J. K., West, N. A., MayerDavis, E. J., Liese, A. D., Marcovina, S. M., D’Agostino, R. B. Jr.,
Hamman, R. F., and Dabelea, D. (2010). Inflammatory markers are increased in youth with type 1 diabetes: The SEARCH case-control study. J. Clin. Endocrinol. Metab. 95, 2868-2876.

Strong, J. P., Malcom, G. T., McMahan, C. A., Tracy, R. E., Newman, W. P., Herderick, E. E., and Cornhill, J. F. (1999). Prevalence and extent of atherosclerosis in adolescents and young adults. JAMA 281, 727.

Trigona, B., Aggoun, Y., Maggio, A., Martin, X. E., Marchand, L. M., Beghetti, M., and Farpour-Lambert, N. J. (2010). Preclinical noninvasive markers of atherosclerosis in children and adolescents with type 1 diabetes are influenced by physical activity. J. Pediatr. 157, 533-539.

Urbina, E. M., Williams, R. V., Alpert, B. S., Collins, R. T., Daniels, S. R., Hayman, L., Jacobson, M., Mahoney, L., MietusSnyder, M., and Rocchini, A. (2009).
Noninvasive assessment of subclinical atherosclerosis in children and adolescents: recommendations for standard assessment for clinical research: a scientific statement from the American Heart Association. Hypertension 54, 919.

Valsania, P., Zarich, S. W., Kowalchuk, G. J., Kosinski, E., Warram, J. H., and Krolewski, A. S. (1991). Severity of coronary artery disease in young patients with insulin-dependent diabetes mellitus. Am. Heart J. 122, 695-700.

Vijayaraghavan, K. (2010). Treatment of dyslipidemia in patients with type 2 diabetes. Lipids Health Dis. 9, 144.

Wadwa, R. P. (2006). Cardiovascular disease risk in youth with diabetes mellitus. Rev. Endocr. Metab. Disord. 7, 197-204.

Zhan, W. W., Chen, Y. H., Zhang, Y. F., Zhu, Y., Lin, Y. Y., Ren, X. P., Li, X. Y., and Liu, Y. P. (2009). Carotid stiffness and microalbuminuria in patients with type 2 diabetes. Endocrine 35, 409-413.
Conflict of Interest Statement: The authors declare that the research was conducted in the absence of any commercial or financial relationships that could be construed as a potential conflict of interest.

Received: 05 May 2011; accepted: 17 September 2011; published online: 11 October 2011.

Citation: Law JR, Patel $S$ and Spagnoli A (2011) Approach to lipid screening as a risk marker for cardiovascular disease in pediatric patients with diabetes. Front. Endocrin. 2:47. doi: 10.3389/fendo.2011.00047

This article was submitted to Frontiers in Pediatric Endocrinology, a specialty of Frontiers in Endocrinology.

Copyright (C) 2011 Law, Patel and Spagnoli. This is an open-access article subject to a non-exclusive license between the authors and Frontiers Media SA, which permits use, distribution and reproduction in other forums, provided the original authors and source are credited and other Frontiers conditions are complied with. 